\title{
Car-Sharing auf der Standspur?
}

\begin{abstract}
In der Bevölkerung besteht laut Umfragen reges Interesse an Car-Sharing. Auch die Politik lobt dieses Beispiel für öko-effiziente Dienstleistungen. Trotzdem stagnieren die Mitgliederzahlen von Car-Sharing-Unternehmen. Ein Grund ist das unterschiedliche Selbstverständnis der Car-Sharing-Anbieter, das ein einheitliches Auftreten und die Interessenbündelung der insgesamt umsatzschwachen Branche erschwert. Um den Weg in Richtung Massenmarkt zu meistern, ist es an der Zeit die entwickelten Innovationen umzusetzen. Professionalisierung und Modernisierung weisen den Weg auf die Überholspur.
\end{abstract}

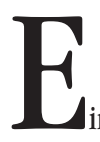

$\mathrm{E}$ Von Siegfried Frick, Andreas Knie und Stefan Reindl Kunden und ein hochgerechnetes Marktvolumen von 12 Milliarden Mark. Dies ermittelte die erste repräsentative Bevölkerungsbefragung zum Thema Car-Sharing in Deutschland für die Car-Sharing-Variante des ,Kilometer-Leasing: Auto auf Abruf" (1). Rund 18,8 Prozent der Befragten zeigten konkretes Interesse am ,Auto auf Abruf“. Die Interessierten kamen aus allen sozialen Schichten und Einkommensgruppen, wobei Frauen, Singlehaushalte und Bewohner von Ballungszentren überdurchschnittlich interessiert waren. Das Marktpotenzial geht also weit über eine soziokulturelle Nische hinaus. Deutlich wurde auch, dass es in der Bevölkerung erhebliche zusätzliche, derzeit nicht befriedigte Mobilitätsbedürfnisse gibt, die durch ein solches automobiles Konzept - sozusagen als Substitut für den Zweit- und Drittwagen - bedient werden könnten.

\section{Ernüchternde Realität}

Ein riesiges Marktpotenzial, öffentliche Anerkennung und höchste politische Weihen - stehen also alle Zeichen auf grün? Die Realitäten hinter den Schlagzeilen sehen anders aus. Die Mitgliederzahlen der organisierten Car-Sharing-Organisationen stagnieren in Deutschland seit Jahren. Es sind immer noch nicht mehr als 37.000 CarSharer. Von den über 80 Einrichtungen in 200 Städten Deutschlands, die Car-Sharing betreiben, hat die Hälfte nicht einmal einen Umsatz von 100.000 Mark.

Vom Durchbruch kann also keine Rede sein. Der bislang ausgebliebene Erfolg ist in erster Linie hausgemacht: Kaum ein Unternehmen in der CarSharing Szene wird als Wirtschaftsunternehmen professionell geführt. Personengesellschaften und Vereine dominieren die Landschaft, die Unternehmens- und Projektphilosophien gehen weit auseinander und reichen vom fundamentalen ,Jeder gefahrene Autokilometer ist einer zuviel" bis zum Bestreben, das Car-Sharing als Kurzzeitvermietung zu einer modernen Dienstleistung auszubauen. Der Bundesverband Car Sharing (BCS) hat es da mit einer wirksamen Interessenvertretung schwer und kann die Gegensätze maximal moderieren, aber nicht substanziell einebnen und wirkungsvoll bündeln.

Die StattAuto AG in Berlin und Hamburg demonstriert zur Zeit, wie der Weg in eine professionelle Dienstleistung aussehen kann, aber auch wie beschwerlich dieser ist. Nach der 1998 erfolgten Fusion zwischen den GmbHs in Berlin und Hamburg musste man nach einem Jahr feststellen, dass unter dem einheitlichen Firmendach die zwei Firmen munter fortlebten, weil im branchenüblichen Glauben an die Gutmütigkeit der Aufbau von Controlling- und Berichtssystemen nur nachlässig betrieben wurde. Aber man konnte reagieren und tat etwas, was bislang völlig unüblich gewesen war: Man trennte sich von Kollegen, zentralisierte die Verwaltung und startete ein konsequentes Modernisierungsprogramm.

Diese neue unternehmerische Performance ist dringend notwendig, denn die Ergebnisse zahlreicher wissenschaftlicher Untersuchung belegen: Die Car-Sharing-Teilnehmer kommen in die Jahre! Es fehlt der Nachwuchs. Zur Zeit ist der mehrheitlich männliche Durchschnittsteilnehmer knapp 40 Jahre alt, hat einen Hochschulabschluss und verdient überdurchschnittlich. Beim Start des organisierten Car-Sharing in Deutschland vor nunmehr zehn Jahren betrug das Durchschnittsalter 30 Jahre. Car-Sharing-Mitglieder sind Menschen, die durch die Umwelt- und Friedensbewegung der 70er und 80er Jahre geprägt sind. Solche Sozialisationsinstanzen stehen heute nicht mehr zur Verfügung. Aus dieser Zeit stammt auch die Vielfältigkeit und Buntheit der Erscheinungsformen des Car-Sharing.

Was vor zehn Jahren noch als Zeichen kreativen Aufbruchs gewertet wurde, ist mittlerweile ein Zeichen der Schwäche. Die Organisationen, die so unterschiedliche Namen wie StattAuto, StadtAuto, Stadtmobil oder Mobilkonzept tragen, verfügen über keine einheitliche Dachmarke, die für Kunden einen Wiederkennungswert darstellt und Qualitätsstandards sichert. Mitgliedsbedingungen und Tarifsysteme sind so vielfältig wie die unterschiedlichen Rechtsformen. Die verwendete Technik bei Fahrzeugaufbewahrung und -übergabe ist ebenfalls vielfältig, meist altmodisch und unpraktisch, denn sie unterscheidet sich in der Regel von Stadt zu Stadt. Die Eintrittsbarrieren für die CarSharing-Nutzer (Einlage, Aufnahmegebühr und Monatsbeitrag) sind - noch aus den Zeiten einer bewussten Anti-Autohaltung stammend - zum Teil sehr hoch. Ein Wettbewerb zwischen den einzelnen Organisationen findet nicht statt, die markterschließende Bündelung der Kräfte aber auch nicht. Ähnlich der früheren Gebietsaufteilung im Energiemarkt haben sich die einzelnen Organisationen ihre Marktgebiete untereinander fein aufgeteilt.

Unterstïtzt wird von den Mitgliedern daher lieber nur die Idee des Car-Sharings und weniger die Dienstleistung selbst. In gut geführten Car-Sharing-Organisationen macht das durchschnittliche Car-Sharing-Mitglied einen Umsatz von unter 1000 Mark pro Jahr. Wenn man sich jetzt vor Augen hält, dass die flächendeckende Bereitstellung der Dienstleistung an über 50 Stationen wie alleine in Berlin sehr kostenintensiv ist, wird klar, dass die Ertragskraft der Unternehmen nicht sehr hoch sein kann.

\section{Car-Sharing am Scheideweg}

Der fehlende gemeinsame Marktauftritt, suboptimaler Komfort und Bequemlichkeit und die hohen Eintrittsbarrieren verhindern das Kundenwachstum. Die kleinteilige Organisationsstruktur verhindert die Ausnutzung von ökonomischen Größenvorteilen. Folglich drücken die Kosten, die Deckungsbeiträge sind gering, für technologische Innovationen und pfiffiges Marketing fehlt das Geld. Auch Löhne und Gehälter bleiben niedrig, qualifizierte Kräfte bleiben aus, die Qualität der Dienstleistung kann nicht ge- 
steigert werden. Aus diesem Teufelskreis ist nur schwer auszubrechen. Die einzelnen Unternehmen sind mit ihren Gründern tief in die örtlichen Politiknetzwerke eingeflochten, die bei Bedarf als eine Art Schutzschild gegen jede Art von Veränderungen in der Geschäftspraxis eingesetzt werden können.

Was dem Car-Sharing in Deutschland am Beginn des 21. Jahrhunderts fehlt, ist ein angemessenes „Format" und ein Professionalisierungs- und Modernisierungsschub - wie er in der Schweiz bislang erfolgreich vollzogen wurde (2). Mobility Car-Sharing Schweiz hat den Weg der Konzentration und Professionalisierung beschritten. Das Unternehmen erreicht eine Marktdurchdringung, von der die deutsche Car-Sharer-Szene derzeit nur träumen kann - auch wenn zum Beispiel die siedlungsstrukturellen Voraussetzungen in der Schweiz anders sind. Mobility allein hat fast genauso viele Kunden wie alle deutschen Car-Sharer zusammen - obwohl Deutschland rund zehnmal größer ist als die Schweiz! Der große Sprung in den Kundenzahlen kam freilich erst, nach dem der „Kantönligeist“ überwunden und eine einzige Marketing- und Technikplattform als Organisation etabliert wurde.

\section{- Aufbruch aus trägen Strukturen?}

Natürlich wird auch in Deutschland an der Überwindung der trägen und nicht marktfähigen Strukturen an vielen Ecken und Enden gearbeitet und experimentiert. Zwei Beispiele seien hier erwähnt:

Die Berliner StattAuto AG hat mit Partnern aus Industrie und Wissenschaft eine Forschungs- und Entwicklungstochter namens Choice GmbH (3) gegründet. Sie soll neue Produkte entwickeln, die die chronische Ertragsschwäche ausgleichen. Zukünftig werden alle Formen von Autoverfügbarkeiten, also von wenig Auto wie im klassischen Car-Sharing bis sehr viel Auto wie beim konventionellen Leasing, unter einem Dach vertrieben. Die Kunden können nun wählen, wie viel „Prozent“ Auto sie nutzen wollen. Die EDV wird grundlegend neu aufgebaut und eine Verknüpfung zu Angeboten anderer Verkehrsdienstleister hergestellt, die verkehrsträgerübergreifende $\mathrm{Ab}$ rechnungsformen und Fahrtenplanung ermöglicht. Aber dieses Modernisierungsprogramm läuft keineswegs als harmonisches Projekt. Gerade in Berlin hat die Nischenwirtschaft noch viele Freunde.

Im Nachgang zu einer Initiative des Forschungsministeriums zum Thema öko-effiziente Dienst- leistungen (4) etablierte sich der Versuch einer gemeinsamen Anstrengung von deutschen CarSharing-Organisationen, Mobility Car-Sharing Schweiz sowie von Unternehmen und Organisationen aus dem Automobilhandel. Denn die Interessen sind durchaus kompatibel: Die autohauseigenen Vermietgesellschaften wie Euromobil und Opel-Rent vereinigen in Deutschland bereits heute über 40000 Fahrzeuge, die wesentlich als Reparatur- und Unfallersatzfahrzeuge eingesetzt werden. Ihre Auslastung am Wochenende ist aber ungenügend. Umgekehrt ist bei den Car-Sharing-Organisationen am Wochenende und in den großen Ferien der Flottenbedarf am größten, die Zuteilungswahrscheinlichkeit zum Ärger der Kunden aber am geringsten. Und: Mit den Autohäusern als Partner könnten neue Kundengruppen für Car-Sharing erschlossen werden - ein einheitlicher Marktauftritt und eine moderne Technikplattform vorausgesetzt. Die MVG Mobilitätsverbundgesellschaft $\mathrm{GmbH}$ wurde gegründet, um dies professionell anzugehen. Alsbald wurde die MVG aber wieder aufgelöst. Das mit viel Zeitaufwand und hohen Kosten betriebene Projekt scheiterte an den unterschiedlichen Kulturen und daran, dass für alle der Handlungsdruck wohl noch nicht groß genug war, um Organisationsstrukturen zu verändern, Kapital zu mobilisieren und gemeinsam ein neues Geschäftsfeld zu erschließen.

\section{- Innovationskraft entscheidet}

Gute Ideen für öko-effiziente Dienstleistungen und ein erhebliches Marktpotenzial allein bewirken also noch keine Markterschließung. Die dem Car-Sharing zugeschriebene umwelt- und verkehrspolitische Bedeutung bleibt aus, wenn die Organisationen nicht in der Lage sind, das Marktpotenzial jenseits der lokalen Nische zu erobern. Strategische Allianzen zur Markterschließung sind - auch wenn die Interessen kompatibel sind - fragile Gebilde, solange sie sich nicht auf mittlere Frist in Mark und Pfennig auszahlen. Der Einstieg in den Massenmarkt der automobilen Kurzzeitmiete erfordert eine einheitliche Mobilitätsdachmarke, lückenlose Flächenabdeckung des Angebots, eine überregional durchgängige Nutzungsmöglichkeit sowie mehr Komfort und Bequemlichkeit für die Kunden. Von Nöten sind außerdem günstigere Tarife, eine neue technologische Plattform auf der Basis modernster Verkehrsleittechnik (Telematik), die Vernetzung der privaten und öffentlichen Verkehrsträger, die Ansprache neuer Kundengrup- pen durch neue Vertriebsallianzen und sehr intensive Marketinganstrengungen, um die Vorteile einer vernetzten Mobilität umsatzwirksam werden zu lassen.

Die Konzeptphase des Nachdenkens über ökoeffiziente Dienstleistungen ist längst beendet. Nun steht die Umsetzung von Innovationen an. ÖkoEffizienz muss in Business-Pläne überführt werden, Nachhaltigkeit muss auch für Banken attraktiv werden. Sollte dies nicht gelingen, weil das Agieren in der Nische komfortabler ist als die raue Welt des Massengeschäfts, verliert das CarSharing seinen Bonus und bleibt eine Fußnote der Verkehrsgeschichte.

\section{Anmerkungen}

(1) Vgl. Frick, S./ Diez, W./ Reindl, S.: Marktchancen für das $\mathrm{Kfz}$-Gewerbe durch öko-effiziente Dienstleistungen. Forschungsbericht Nr. 15/1998 des IFA-Instituts für Automobilwirtschaft, Nürtingen/Geislingen 1998 und

Diez, W./ Frick, S./ Reindl, S.: Kilometer-Leasing als innovative Mobilitätsdienstleistung. In: Internationales Verkehrswesen, 51 (1999) 8, S. $383-386$.

(2) Vgl. im Internet unter www.mobility.ch

(3) Vgl. im Internet unter www.choice.de sowie Canzler,

W./ Knie, A.: Möglichkeitsräume: Grundrisse einer modernen Mobilitäts - und Verkehrspolitik, Wien 1998.

(4) Vgl. Frick, S./ Gaßner, R./ Hinterberger, F./ Liedtke, C.: (Hrsg.): Öko-effiziente Dienstleistungen als strategischer Wettbewerbsfaktor zur Entwicklung einer nachhaltigen Wirtschaft. Werkstattbericht Nr. 36 des Instituts für Zukunftsstudien und Technologiebewertung, Berlin 1999 sowie

Klemmer, P./ Hinterberger, F. (Hrsg.): Öko-effiziente Dienstleistungen. Berlin u.a. 1999.

\section{Die Autoren}

Siegfried Frick ist Analyst/Manager im Automotive Research Team der Investmentbank WestLB Panmure, Düsseldorf.

Kontakt: Tel. 0211/ 826-4988, E-Mail: siegfried. frick@westlbpanmure.com

Dr. Andreas Knie ist Geschäftsführer der Choice $\mathrm{GmbH}$, Berlin, wissenschaftlicher Angestellter des WZB Berlin sowie Hochschullehrer an der TU Berlin. Kontakt: Tel. 030/ 2314910,

E-Mail: knie@choice.de

Stefan Reindl ist wissenschaftlicher Mitarbeiter am

Institut für Automobilwirtschaft (IFA), Geislingen. Kontakt: Tel. 07331/ 949530,

E-Mail: s.reindl@ifa-info.de 
(c) 20I0 Authors; licensee IÖW and oekom verlag. This is an article distributed under the terms of the Creative Commons Attribution Non-Commercial No Derivates License (http://creativecommons.org/licenses/by-nc-nd/3.o/), which permits unrestricted use, distribution, and reproduction in any medium, provided the original work is properly cited. 